\title{
Fabrication of ZnO nanorods for optoelectronic device applications
}

\author{
R Chakraborty ${ }^{1}, \mathrm{U}$ Das $^{1 *}$, D Mohanta $^{1,2}$ and A Choudhury ${ }^{1,3}$ \\ ${ }^{1}$ Nanoscience Laboratory, Department of Physics, Tezpur University, P.O. Napaam, Tezpur-784 028, Assam, India \\ ${ }^{2}$ Laboratory for Molecular Scale Engineering, Electrical and Computer Engineering, \\ University of Wisconsin-Madison, WI-53706, USA \\ ${ }^{3}$ Gauhati University, Gopinath Bordoloi Nagar, Guwahati-781 014, Assam, India
}

E-mail : upam2005@gmail.com

\begin{abstract}
Hydroxyl free zinc oxide nanorods have been synthesized by a catalyst free surfactant based one-step solid state reaction process. The powder X-ray diffraction studies reveal well defined wurtzite peaks due to crystalline $\mathrm{ZnO}$, while optical absorption spectra represent prominent exciton absorption and remarkable blueshift in the onset of absorption. As predicted by transmission electron microscopy, the $\mathrm{ZnO}$ nanorods are $\sim 100 \mathrm{~nm}$ long and of $\sim 20 \mathrm{~nm}$ dia. Further, luminescence aspects of such nanorods are studied for possible deployment in optoelectronics devices.
\end{abstract}

Keywords : Nanorods, optoelectronics, luminescence.

PACS Nos. : $61.46 . \mathrm{Km}, 81.07 . \mathrm{Wx}, 78.67 .-\mathrm{n}, 81.07 .-\mathrm{b}$

\section{Introduction}

Recently, one-dimensional semiconductor systems such as nanorods, nanotubes, nanoneedles, nanowires etc. have gained enormous research interests as they are considered to be potential candidates for microelectronics, photonics, and sensing applications [1-8]. Till date, extensive literature is available highlighting fabrication details/methods e.g., chemical vapor deposition [9-12], arc discharge [13], laser ablation [14], template based method [15,16] etc.

Zinc oxide ( $\mathrm{ZnO})$, a II-VI binary-compound semiconductor, has a wurtzite structure with a direct band gap of $3.37 \mathrm{eV}$ at room temperature. The wide band gap along with large exciton binding energy ( 60 meV) makes $\mathrm{ZnO}$ as an attractive candidate for UV sensors/detectors [17]. Compared to the bulk-ZnO, nano-ZnO exhibits extremely large excition binding energy and therefore, excitions in the later case are much more stable 
at room temperature $\left(k_{\beta} T \sim 25 \mathrm{meV}\right)$. As an alternative candidate of nanoelectronics, it was reported that $p$-type conductivity can be induced in natural hexagonal crystal structure of $\mathrm{ZnO}$ nanowire and was ascribed to the presence of oxygen vacancies. Also, the combined magnetic and transport properties as well as high Curie temperature etc. have made doped-ZnO a potential candidate for spintronic applications $[18,19]$. Further, polarized light emission from $\mathrm{ZnO}$ nanorods has been demonstrated recently [20].

In this letter, we report one-step synthesis of $\mathrm{ZnO}$ nanorods adopting a cost effective, catalyst free solid state reaction route. The as prepared samples were characterized by X-ray diffraction (XRD), transmission electron microscopy (TEM) and absorption spectroscopy. In addition, photoluminescence studies are performed to reveal optoelectronic properties.

\section{Experimental details}

$\mathrm{ZnO}$ nanorods were synthesized at room temperature by a simple and convenient physico-chemical reaction. The source material was a mixture of zinc acetate dihydrate (ZAD) $\left[\mathrm{Zn}\left(\mathrm{CH}_{3} \mathrm{COO}\right)_{2} \cdot 2 \mathrm{H}_{2} \mathrm{O}\right.$ ], cetyl trimethyl ammonium bromide (CTAB, a cationic surfactant) $\left[\mathrm{C}_{19} \mathrm{H}_{42} \mathrm{BrN}\right]$, and sodium hydroxide flakes in molar ratio $1: 0.5: 3$. Without applying direct pressure, the mixture was ground in a mortar for 1-2 hours. Next, the mixture was transferred to a culture-tube and kept in an ultrasonic bath for one hour. The sonicated mixture was then washed with double distilled water several times. In order to induce oxidation of unreacted species, the as prepared mixture was annealed at temperature $60-80^{\circ} \mathrm{C}$ for 2 hours. Finally, the product was preserved for further experimentation and characterization.

In order to reveal structural morphology, with regard to size, shape distribution and crystallinity the sample was studied by powder diffraction and electron microscopy. The XRD pattern was recorded on a powder sample with a Rigaku D/max-2000

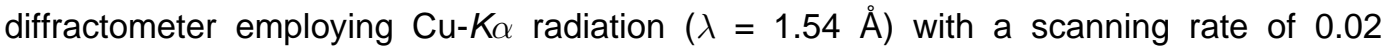
degree/s. The TEM images were taken by employing a JEOL JSM-100 CX microscope working at a beam accelerating voltage of $80 \mathrm{KV}$. Optical properties were investigated by absorption and photoluminescence (PL) spectroscopy. The room-temperature $\mathrm{PL}$ spectra were recorded by a Perkin-Elmer LS-55 spectrophotometer using $\lambda=325 \mathrm{~nm}$ as the excitation wavelength.

\section{Results and discussion}

The structural phase and crystallographic orientation of the as-grown nano- $\mathrm{ZnO}$ samples (Figure 1) were identified by XRD measurements. The diffraction pattern suggests that as-grown nanostructures are polycrystalline in nature with wurtzite crystal phase in consistency with earlier reports [21].

The strongest diffraction (101) peak at $34.51^{\circ}$ implied that the structures were 


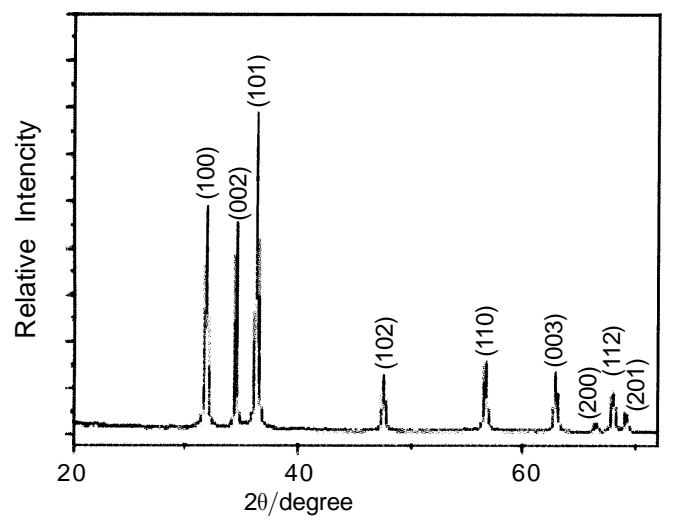

Figure 1. XRD spectra of $\mathrm{ZnO}$ nanorods.

grown with preferred orientation of (101) plane. From the presented data, the crystal lattice parameters are estimated as : $a=3.235 \AA$ and $c=5.189 \AA$, which are close to $a=3.253 \AA$ and $c=5.209 \AA$ of ZnO (JCPDS Card No. 80-00075). In the XRD pattern, no characteristic peak due either to impurities or unreacted species like $Z n$, CTAB or $\mathrm{Zn}(\mathrm{OH})_{2}$ is observed. This results actually indicates the formation of hydroxyl free, wurtzite $\mathrm{ZnO}$ phase.

The absorption spectrum is represented in Figure 2. Single sharp exciton absorption peak was noticed at $\sim 350 \mathrm{~nm}$ owing to $1 s-1 s$ transition states. Also, narrow tailing in the OAS ensures us that there is adequate suppression in the degree of

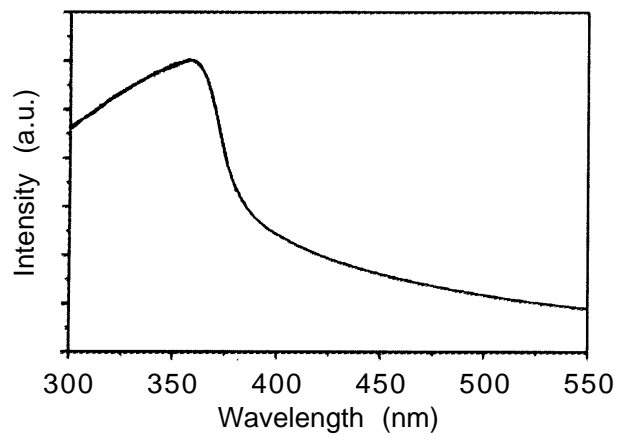

Figure 2. UV-VIS optical absorption spectra of $\mathrm{ZnO}$ nanorods.

inhomogeneity due to improved and organizational growth of particles while synthesis was in progress.

A clear evidence of the formation of elongated $\mathrm{ZnO}$ nanostructures is being depicted in Figure 3. The Figure shows an isolated $\mathrm{ZnO}$ nanorod, which has a typical length of $\sim 100 \mathrm{~nm}$ and of dia $\sim 20 \mathrm{~nm}$. Interestingly, we notice that the nanorods have deviated from the ideal solid-cylindrical shape. In other words, two extreme ends of the nanorods have smaller dia compared the middle parts and therefore, resembling commercial ball-pen like structures. 


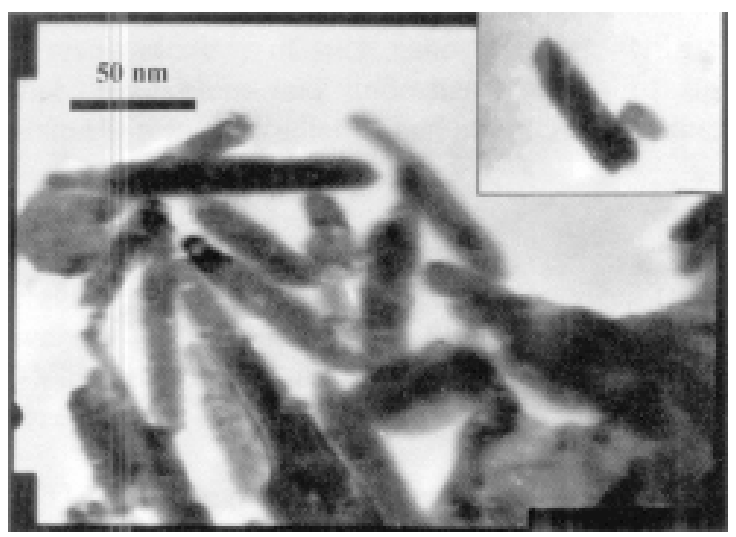

Figure 3. TEM image of $\mathrm{ZnO}$ nanorods.

Since the hydroxides are decomposed by a strong heat of the reaction to produce oxides in the reaction process, organization and assimilation of particles would be slow during initiation and termination in the formation of $\mathrm{ZnO}$ nanorods. In reference to the mechanism of growth process first, $\mathrm{Zn}(\mathrm{OH})_{2}$ was formed due to compositional formed mixing facilitated by solid state reaction and finally, $\mathrm{ZnO}$ is produced due to gradual decomposition owing to strong heat of reaction.

The photoluminescence studies are presented in Figure 4. The $325 \mathrm{~nm}$ line of

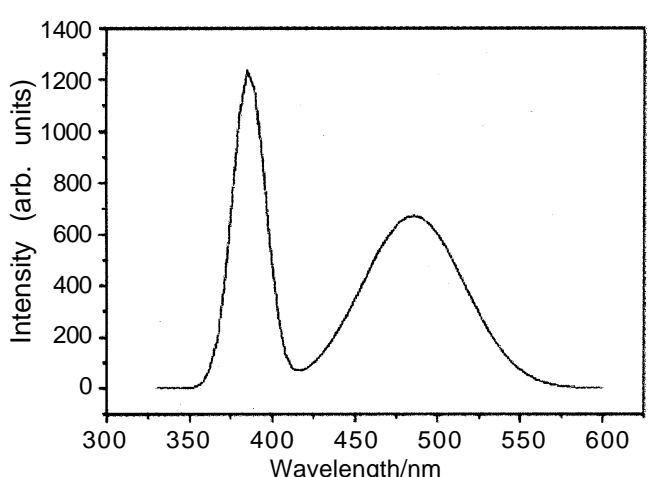

(a)

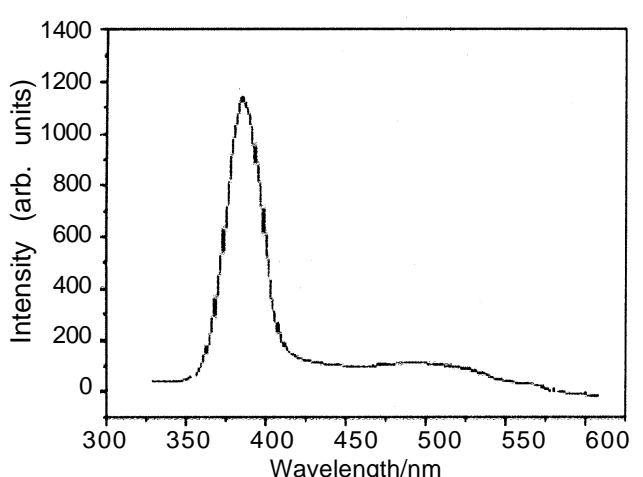

(b)

Figure 4. Room temperature PL spectra of (a) nano and (b) bulk $\mathrm{ZnO}$.

Xe-lamp was used as the excitation source and the data were collected by a computer controlled standard monochromator based photodetection system. The sharp peak at $\sim 375 \mathrm{~nm}$ shows the near-band-edge (NBE) emission. This is generally associated with the excitonic transitions [22], which has been observed between 378-385 $\mathrm{nm}$ by earlier workers [23-26]. A second broad band peak $\sim 485$ is noticeable only in nano-ZnS sample and is ascribed to the trap related emission. The trap emission is associated with the nonradiative centers, generally inherent in nanostructures owing to extremely large surface-to-volume ratio. 
Previously, the presence of broader PL band in the visible green region was attributed to the recombination of photogenerated holes with the singly ionized oxygen vacancies [22]. Also, it was reported that the defect induced emission can be suppressed by choosing a suitable substrate that avoids lattice mismatch to a large extent [27]. In our powder sample system, there is no such reason that can control trap emission. The symmetric nature of the green emission with FWHM $\sim 105 \mathrm{~nm}$ reflects uniform roughness all throughout the nanorods. Highlighting polarized light emission characteristics, tunability of near band edge and defect emission was demonstrated by earlier workers [20]. In our case, strong and symmetric response of both the emission features of PL also support organized growth of quality nanorods. Considering absorption and emission spectra we have estimated binding energy of excitons $E_{b} \sim 120 \mathrm{meV}$. The calculated value is close to the value estimated by other workers [28].

\section{Conclusion}

In summary, we have described a one step solid state synthesis of $\mathrm{ZnO}$ nanorods. The method described here is simple, robust and cost effective for large scale production. One dimensional nanorods of length at least five times more than the base dimension were produced. The powder diffraction study provides signature of hexagonal wurtzite structure with (101) as preferred orientation. Symmetric nature of trap-luminescence, centered at $\sim 485 \mathrm{~nm}$ suggests uniform smoothness over the nanorod surfaces. The binding energy of our $\mathrm{ZnO}$ nanorod sample was predicted to be $\sim 120 \mathrm{meV}$. Temperature dependent and time resolved study of such nanostructures, will help further, to explore and understand nature of light emission in terms of radiative and nonradiative transitions.

\section{Acknowledgments}

The authors thank Department of Chemical Sciences, Tezpur University and SAIF, NEHU, Shillong for extending UV-VIS spectroscopy and TEM facility, respectively.

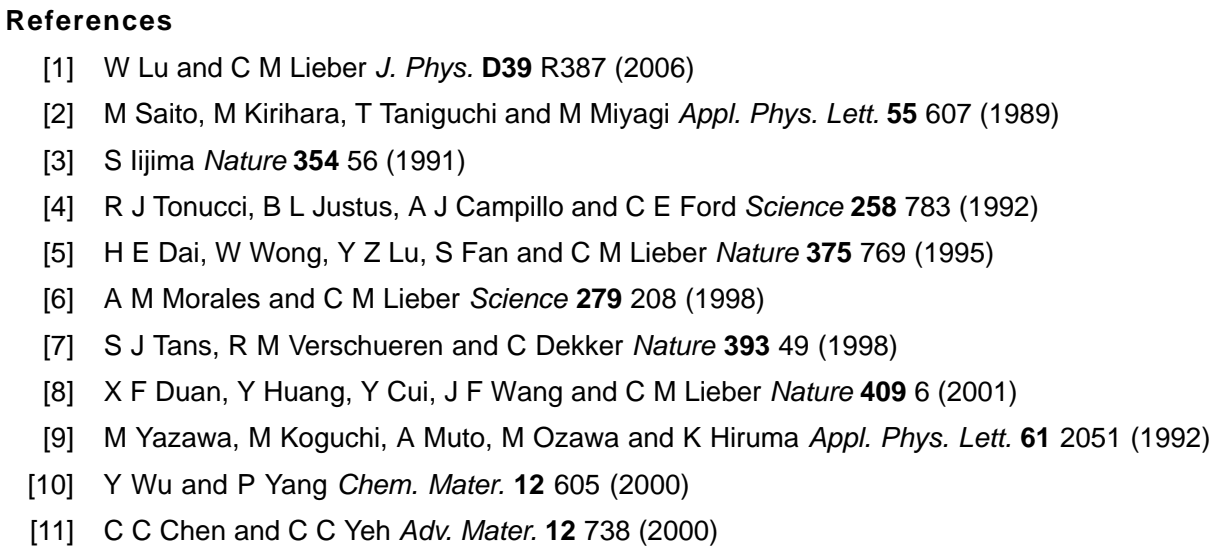


[12] Z G Bai, D P Yu, H Z Zhang, Y Ding, X Z Gai, Q L Hang, G C Xiong and S Q Feng Chem. Phys. Lett. 303 311 (1999)

[13] Y C Choi, W S Kim, Y S Park, S M Lee, D J Bae, Y H Lee, G-S Park, W B Choi, N S Lee and J M Kim Adv. Mater. 12746 (2000)

[14] (a) X F Duan and C M Lieber Adv. Mater. 12298 (2000); (b) A M Morals and C M Lieber Science 279208 (1998)

[15] (a) M H Huang, A Choudrey and P Yang Chem. Commun. 121063 (2000); (b) J Zhu and S Fan J. Mater. Res. 141175 (1999)

[16] Y Li, G W Meng, L D Zhang and F Philipp Appl. Phys. Lett. 762011 (2000)

[17] S P Chang, S J Chang, Y Z Chiou, C Y Lu, T K Lin, Y C Lin, C F Kuo and H M Chang J. Elec. Chem. Soc. $154 \mathrm{~J} 209$ (2007)

[18] B K Roberts, A B Pakhomov, V S Shutthanandan and K M Krishnan J. Appl. Phys. 97 10D310 (2005)

[19] T S Herng, S P Lau, a_S F Yu, H Y Yang and X H Ji J S Chen, N Yasui and H Inaba 99086101 (2006)

[20] N E Hsu, W K Hung and Y F Chen J. Appl. Phys. 964671 (2004)

[21] S J Kwon, J-H Park and J-G Park Appl. Phys. Lett. 87133112 (2005)

[22] L Spanhel and M A Anderson J. Am. Chem. Soc. 1132826 (1991)

[23] L E Greene, M Law, J Goldberger, F Kim, J C Johnson, Y Zhang, R J Saykally, P Yang and Angew Chem. Int. Ed. 423031 (2003)

[24] J W P Hsu, D R Tallant, R L Simpson, N A Missert and R G Copeland Appl. Phys. Lett. 88252103 (2006)

[25] W M Kwok, A B Djurisic, Y H Leung, W K Chan and D L Phillips Appl. Phys. Lett. 87223111 (2005)

[26] K Vanheusden, W L Warren, C H Seager, D R Tallant, J A Voigt and B E Gnade J. Appl. Phys. 797983 (1996)

[27] H Chik, J Liang, S G Cloutier, N Kouklin and J M Xu Appl. Phys. Lett. 843376 (2004)

[28] H P He, Z Z Ye, S S Lin, H P Tang, Y Z Zhang, L P Zhu, J Y Huang and B H Zhao J. Appl. Phys. 102013511 (2007) 